\title{
Función tiroidea en pacientes pediátricos con enfermedad renal crónica
}

\author{
FRANCISCA UGARTE P. ${ }^{1}$, GIANNINA IZQUIERDO C. ${ }^{2}$, PEDRO ZAMBRANO O. ${ }^{3}$, \\ GERARDO FASCE P. ${ }^{4}$, VIOLA PINTO S. ${ }^{3}$, ALEJANDRA CORTÉS B. ${ }^{3 A}$ \\ 1. Endocrinóloga Infantil, Unidad de Endocrinología, Hospital Exequiel González Cortés. \\ 2. Pediatra, Hospital Exequiel González Cortés \\ 3. Nefrólogo, Unidad de Nefrología, Hospital Exequiel González Cortés. \\ 4. Médico, Magister en Epidemiología Clínica, Universidad de Chile. \\ A. Enfermera Universitaria.
}

\begin{abstract}
Thyroid Function in Pediatric Patients with Chronic Renal Failure

Objective: To characterize the thyroid function in mild (L), moderate (M), hemodialysis (HD), peritoneal dialysis (PD), chronic renal failure (CRF) and post kidney transplant (TX). Method: 46 children between $9.3 \pm 3.7$ years-old with CRF (10 mild (L), 10 moderate $(M), 10$ peritoneodialysis $(\mathrm{PD}), 6$ hemodialysis (HD), 10 transplants (TX)) were evaluated. Basal total T4 and free T3, TRH test (TSH at 0-30-60 min), creatinine, BUN, creatinine clearance and anthropometric parameters were measured. The statistics analysis included Anova Test to compare group results and correlation coefficients for studied variables. Results: Basal thyroid hormone levels were normal in all groups and no differences between groups (except higher TSH in $\mathrm{L}(\mathrm{p}<0.01)$ ) were found. TRH test response was prolonged on $\mathrm{L}, \mathrm{M}, \mathrm{PD}$ and HD and deficient in TX, except 3 TX patients who had normal TRH response, all using Tacrolimus, Micofenolate and Prednisone on alternate day treatment versus the remaining TX who where on Cyclosporine or Azathioprine, Micofenolate and continuous corticoid regimen. Prolonged TRH response correlates with creatinine $(p<0.001)$ and creatinine clearance $(p<0,01)$. Conclusions: Basal thyroid hormones were normal in all groups. TRH test response was predominantly prolonged in $\mathrm{L}, \mathrm{M}, \mathrm{PD}$ and $\mathrm{HD}$, suggesting adaptative phenomena at tertiary level, and correlates with renal function. TX patients had deficient TRH response, suggesting hypofisial dysfunction.

(Key words: TRH test, thyroid, chronic renal failure, children).

Rev Chil Pediatr 2008; 79 (3): 259-266
\end{abstract}

\section{RESUMEN}

Objetivo: Caracterizar la función tiroidea y la respuesta a test de TRH (thyroid releasing hormone), en niños con enfermedad renal crónica (ERC) leve (L), moderada (M), peritoneodiálisis (PD), hemodiálisis

Trabajo recibido el 28 de febrero de 2008, aceptado para publicación el 14 de abril de 2008.

Correspondencia a:

Francisca Ugarte $P$.

E-mail: ugartepalacios_fca@hotmail.com 
(HD) y trasplantados renales (TX). Pacientes y Método: Se estudiaron 46 pacientes con ERC (10 L, $10 \mathrm{M}$, $10 \mathrm{PD}, 6 \mathrm{HD}$ y $10 \mathrm{TX}$ ), 9,3 $\pm 3,7$ años. Se midió $\mathrm{t} 4 \mathrm{t}, \mathrm{t} 41$, t3t, t31, TBG basal y test de TRH (TSH a 0, 30 y $60 \mathrm{~min}$ ). Se evaluó función renal, antropometría y se consignó tratamiento inmunosupresor (IS) en el grupo TX. Se utilizó anova para comparar los resultados entre los grupos y coeficiente de correlación para las variables estudiadas. Resultados: Los valores basales de hormonas tiroideas fueron normales en todos los grupos, sólo TSH fue significativamente mayor en $\mathrm{L}$ aunque dentro del rango normal $(\mathrm{p}<0,01)$. La respuesta al test de TRH fue predominantemente prolongada en L, M, PD y HD y deficiente en TX; los 3 pacientes TX con tacrolimus, micofenolato y prednisona en días alternos tuvieron respuesta normal a diferencia del resto TX que recibían prednisona continua, ciclosporina y micofenolato. La prolongación de respuesta a TRH se correlacionó con creatininemia, BUN y clearance de creatinina $(p<0,01)$. Conclusiones: Los niveles de hormonas tiroideas basales se encuentran normales en todos los grupos de ERC. La respuesta a TRH fue predominantemente prolongada en $\mathrm{L}, \mathrm{M}, \mathrm{PD}$ y $\mathrm{HD}$, demostrando un fenómeno adaptativo a nivel terciario del eje hipotálamo-hipofisis-tiroides. Los TX presentan una respuesta mayoritoriamente deficiente a TRH, sugerente de disfunción hipofisiaria, la que podría estar relacionada con el tipo de tratamiento inmunosupresor y al uso de corticoides en días contínuos.

(Palabras clave: Test de TRH, tiroides, enfermedad renal crónica, niños)

Rev Chil Pediatr 2008; 79 (3): 259-266

\section{Introducción}

Se han descrito múltiples alteraciones de la glándula y función tiroidea en pacientes con enfermedad renal crónica (ERC). Los estudios publicados, describen disminución en los niveles de T3 y T4, total y libre (T3T, T3L, T4T, $\mathrm{T} 4 \mathrm{~L}$ ), con valores normales de TSH, tanto en pacientes con ERC en tratamiento convencional, hemodiálisis (HD) como en peritoneodiálisis $(\mathrm{PD})^{1-3}$. Los mecanismos que estarían involucrados en estas alteraciones serían una disminución de la conversión periférica de $\mathrm{T} 4$ a T3, alteración en la unión de $\mathrm{T} 4$ con la proteína trasportadora de hormonas tiroideas (TBG, thyroid binding globulin) por inhibidores urémicos y/o alteraciones a nivel hipotálamo hipofisiario ${ }^{3-5}$. Por otra parte, en pacientes con ERC sometidos a trasplante renal, existiría normalización de los niveles de hormonas tiroideas post trasplante ${ }^{6}$.

Pasqualini y cols ${ }^{5}$, estudiaron 9 niños con ERC en tratamiento de reemplazo (PD y HD), midiendo los niveles de hormonas tiroideas así como la respuesta al test de TRH (thyroid releasing hormone); sólo 1 paciente tenía hipotiroidismo primario y 8 tenían valores normales de TSH con una respuesta a TRH alterada (7 prolongada y 1 deficiente), planteando que una alteración a nivel hipotálamo hipofisiario podría explicar estos resultados. $\mathrm{Lim}^{7}$, planteó que los fenómenos descritos serían adaptativos a la ERC y que ayudarían a mantener un estado basal de bajo gasto metabólico con el fin de disminuir la pérdida proteica y la desnutrición secundaria. Por otra parte, en pacientes pediátricos trasplantados se recuperan los valores basales pero presentan una respuesta deficiente al estímulo lo que según los autores sería atribuible a uso de corticoides ${ }^{6}$.

Los trabajos publicados de función tiroidea en niños con insuficiencia renal crónica son escasos, presentan resultados controvertidos y la mayoría fueron realizados hace más de una década en un número reducido de pacientes ${ }^{4-6}$. Los tratamientos de la ERC de entonces, no contemplaban apoyo nutricional intensivo, ni adecuación de la diálisis basada en la cinética de la urea y los pacientes presentaban compromiso nutricional y las expectativas de vida eran menores.

El objetivo de este trabajo es caracterizar la función tiroidea basal y del eje hipotálamohipófisis-tiroides mediante prueba de estimulación exógena con TRH en niños con ERC leve (L), moderada (M), en peritoneodiálisis (PD), hemodiálisis (HD) y post trasplante (TX), con manejo actualizado de ERC, controlados en un hospital público de Santiago. 


\section{Pacientes y Método}

Se realizó un estudio prospectivo, descriptivo, observacional donde se estudiaron todos los pacientes con ERC en etapa M, HD, PD y TX menores de 15 años; controlados en la Unidad de Nefrología del Hospital Exequiel González Cortés entre noviembre 2004 y marzo del 2005. El estudio fue aprobado por el comité de ética local y se obtuvo consentimiento informado de los adultos responsables y/o del niño si este era mayor de 14 años.

Se consideraron criterios de exclusión: ERC secundaria a patología oncológica o derivada de sus tratamientos, patologías crónicas asociadas (hepatopatías, cardiopatías, enfermedades respiratorias crónicas, nefropatías perdedoras de proteínas) y patología tiroidea previa a la ERC.

Según clearance de creatinina (CL crea) estimado por Schwartz ${ }^{8}$, los pacientes fueron asignados a los siguientes grupos: 1 (ERC leve) con CL crea entre 79 y $45 \mathrm{ml} / \mathrm{min}$; M (ERC moderada) con CL crea $15-44 \mathrm{ml} / \mathrm{min}$. Los pacientes con ERC severa, CL de crea $<15$ $\mathrm{ml} / \mathrm{min}$, en tratamiento con peritoneodiálisis se denominaron PD y los con ERC en hemodiálisis HD. Finalmente, los trasplantados renales estables, es decir sin antecedentes de rechazo y con creatininemia menor a $1,2 \mathrm{mg} / \mathrm{dl}$ en 3 tomas en los últimos 6 meses ingresaron al grupo TX.

Se consignó edad, sexo, enfermedad causal de ERC, tipo de tratamiento actual y en el grupo TX el tratamiento inmunosupresor que recibía. También se evaluó la condición antropométrica determinando $\mathrm{Z} \mathrm{T} / \mathrm{E}$ y porcentaje de adecuación peso/talla $(\mathrm{P} / \mathrm{T})$ en los menores de 6 años según las tablas del National Center for Health Statistics (NCHS) e índice de masa corporal (IMC) en los mayores de 6 años, según las tablas del Center of Diseases Control $(\mathrm{CDC})^{9}$.

Las determinaciones hormonales de T4T, T4L, T3T, T3L y TBG se realizaron por quimioluminiscencia utilizando kits comerciales DPC, considerando los valores de referencias según edad, señalados en cada kit. El test de TRH se realizó con hormona liberadora de tirotrofina (TRHelea 200, elea, Argentina), inyectando 7 $\mu \mathrm{g} / \mathrm{kg}$ de TRH ev (200 $\mu \mathrm{g}$ valor máximo) y determinando TSH (quimioluminiscencia DPC) basal, 30 minutos y 60 minutos post administración. Se registraron los efectos adversos al test de TRH y medición de presión arterial pre y post test. Se consideró como valor normal TSH basal de 0,5 a $5 \mathrm{uUI} / \mathrm{ml}$ y frente a estimulación con TRH un aumento de TSH ( $\delta$ max) entre 4,5 y $20 \mathrm{uUI} / \mathrm{ml}$ en los primeros 30 minutos, con caída al valor normal a los $60 \mathrm{~min}$. Se consideró exagerada una respuesta con un $\delta \max >20$ $\mathrm{uUI} / \mathrm{ml}$ y prolongada si permanecía elevada a los 60 min o la TSH no disminuyó más de un $40 \%\left(\right.$ deltaTSH$_{0-60} \times 100 /$ delta $\left.\mathrm{TSH}_{0-30}\right)$. Si el $\delta$ max se produjo a los 60 minutos se consideró como una respuesta retrasada. Una respuesta nula o deficiente es aquella con un $\delta \max <4,5$ $\mathrm{uUI} / \mathrm{ml}^{10}$.

Los resultados se expresan como promedios \pm DS y porcentajes. Se señalan los resultados del grupo total ERC y se compararon los resultados por grupo (anova). Se calcularon los coeficientes de correlación entre las siguientes variables: TSH basal, delta TSH 0-30 y 0-60 min, \% TSH 60 vs $30 \mathrm{~min}$, niveles de creatininemia, clearance creatinina, BUN, índice peso/ talla; utilizando R (pearson) o RHO (spearman), según correspondiese. Las correlaciones se catalogaron como débiles si eran menores a 0,5 , moderadas si se encontraban entre 0,51 y 0,74 $\mathrm{y}$ fuertes con valores $\geq 0,75$. Se consideró significativo un valor de $\mathrm{p}<0,05$.

\section{Resultados}

Se encontraban en control 76 pacientes con ERC, de los cuales cumplían criterios de inclusión 46 pacientes. En los 30 pacientes excluidos (6/16 L, 2/12 M, 4/14 PD, 5/11 HD y 13/23 TX) las causas fueron: Síndrome Down (2), hipotiroidismo previo a ERC (2), hiperplasia suprarrenal congénita (1), trasplantados con rechazo agudo o crónico al momento del estudio (10), pacientes en PD o HD con antecedente de TX previo (6), síndrome nefrótico congénito (1), ficha incompleta (5) y abandono de seguimiento (3).

De los 46 pacientes que ingresaron al estudio, 19 eran varones $(41,3 \%)$, la edad promedio fue 9,3 \pm 3,7 años (rango 1,2-15,9 años); 10 tenían ERC L, 10 M, 10 en PD, 6 en HD y 10 
en TX. No hubo diferencias significativas de edad entre los distintos grupos (tabla 1). Las causas más frecuente de ERC fueron la hipoplasia-displasia renal $(26 \%)$ y nefropatía por reflujo (17,3\%) (tabla 2).

En cuanto al estado nutricional, el índice de $\mathrm{P} / \mathrm{T}$ promedio del grupo total de pacientes fue de $108 \pm 21,1 \%$ (rangos entre 54,6-154,7), tenían sobrepeso u obesidad 36,9\% (17/46), 43,4\% (20/46) eran eutróficos y 19,5\% (9/46) desnutridos. Al analizar por grupos (tabla 1 ) no hubo diferencias significativas entre ellos $(p=N S)$.

$\mathrm{Al}$ evaluar la talla se observó que $Z \mathrm{~T} / \mathrm{E}$ del grupo total era de $-1,5 \pm 1,6$ DS y que $35 \%$ (16/ 46) de los pacientes estudiados tenían talla baja $(\mathrm{Z} \mathrm{T} / \mathrm{E}<-2)$. Al analizar por grupos no hubo diferencias estadísticamente significativas $(\mathrm{p}=$ NS) (tabla 1).

Los valores basales promedios de hormonas tiroideas en el grupo total fueron: TSH $(2,7 \pm$ $1,6 \mathrm{uUI} / \mathrm{ml})$, T4T $(11,6 \pm 2,7 \mu \mathrm{g} / \mathrm{dl}) \mathrm{T} 4 \mathrm{~L}(1,5 \pm$ $0,31 \mathrm{ng} / \mathrm{dl})$, T3T $(185,3 \pm 47,54 \mathrm{ng} / \mathrm{dl}), \mathrm{T} 3 \mathrm{~L}$ $(4,78 \pm 1,43 \mathrm{pg} / \mathrm{ml})$ y TBG $(22,07 \pm 4,26 \mu \mathrm{g} / \mathrm{dl})$, encontrándose dentro del rango normal. Sólo 1 paciente, del grupo 1, tenía TSH basal elevado (7,67 uUI/ml, VR 0,7-6,4 uUI/ml). Al analizar por grupos, no hubo diferencias significativas para los valores de T4T, T4L, T3T, T3L, ni TBG: sólo TSH basal fue significativamente mayor en $1(4,43 \pm 1,37 \mathrm{uUI} / \mathrm{ml})$ vs los demás grupos aunque los valores de encontraban dentro del rango normal $(\mathrm{p}<0,001)$ (tabla 3 ).

$\mathrm{Al}$ analizar la respuesta al test de TRH en el grupo total, se observó que 54\% (25/46) de los pacientes presentaba una respuesta prolongada, 28\% (13/46) deficiente, 8,6\% (4/46) normal, $2,1 \%(1 / 46)$ retrasada, $2,1 \%(1 / 46)$ exagerada y $4,3 \%(2 / 46)$ inclasificables. Evaluado por gru-
Tabla 1. Características generales y antropométricas en pacientes con enfermedad renal crónica, por grupos

\begin{tabular}{lcccc}
\hline Grupo & $\mathbf{n}$ & $\begin{array}{c}\text { Edad } \\
\text { (Meses) }\end{array}$ & \multicolumn{1}{c}{$\begin{array}{c}\mathbf{Z} \text { T/E } \\
\text { (DS) }\end{array}$} & $\begin{array}{l}\text { IPT } \\
\text { (\%) }\end{array}$ \\
\hline Leve & 10 & 120 & $-0,63(2,05)$ & 111,2 \\
Moderado & 10 & 90,8 & $-2(1,6)$ & 108,9 \\
PD & 10 & 109,9 & $-2,27(0,97)$ & 102,4 \\
HD & 6 & 125,5 & $-0,94(1,9)$ & 96,3 \\
Tx & 10 & 120 & $-1,6(1,04)$ & 116,2 \\
& & $p=N S$ & $p=N S$ & $p=N S$ \\
\hline
\end{tabular}

PD: peritoneodiálisis; HD: hemodiálisis; Tx: trasplantados; p: significancia estadística; NS: no significativa
Tabla 2. Causas de enfermedad renal crónica en el grupo estudiado

\begin{tabular}{lrc}
\hline Diagnóstico & $\mathbf{n}$ & (\%) \\
\hline Hipo-displasia renal & 12 & $(26)$ \\
Nefropatía por reflujo & 8 & $(17,3)$ \\
Síndrome hemolítico urémico & 7 & $(15,0)$ \\
Púrpura de Schonlein Henoch & 4 & $(8,6)$ \\
No precisada & 3 & $(6,5)$ \\
Vejiga neurogénica & 2 & $(4,3)$ \\
Riñón poliquístico & 2 & $(4,3)$ \\
Nefritis intersticial & 2 & $(4,3)$ \\
Otros & 6 & $(13)$ \\
Total & 46 & $(100)$ \\
\hline
\end{tabular}

Tabla 3. Perfil tiroideo basal $(\bar{x} \pm$ DS)

\begin{tabular}{lcccccc}
\hline Grupo & T4T $(\boldsymbol{\mu g} / \mathbf{d l})$ & T4L $(\mathbf{n g} / \mathbf{d l})$ & T3T $(\mathbf{n g} / \mathbf{d l})$ & T3L $(\mathbf{p g} / \mathbf{m l})$ & TBG $(\boldsymbol{\mu} \mathbf{g} / \mathbf{d l})$ & TSH (uUl/ml) \\
\hline Leve & $10,85 \pm 1,83$ & $1,5 \pm 0,24$ & $200,6 \pm 30,6$ & $4,73 \pm 0,92$ & $21,07 \pm 3,35$ & $4,43 \pm 1,37^{*}$ \\
Moderada & $12,62 \pm 2,59$ & $1,69 \pm 0,22$ & $175,1 \pm 47,39$ & $4,79 \pm 1,20$ & $21,02 \pm 4,84$ & $2,96 \pm 1,46$ \\
PD & $11,38 \pm 2,24$ & $1,37 \pm 0,22$ & $172,1 \pm 67,72$ & $5,37 \pm 2,29$ & $24,5 \pm 3,35$ & $2,39 \pm 1,39$ \\
HD & $11,61 \pm 4,57$ & $1,56 \pm 0,55$ & $178 \pm 38,81$ & $4,6 \pm 1,32$ & $23,65 \pm 2,49$ & $1,98 \pm 1,19$ \\
Tx & $11,75 \pm 3,09$ & $1,59 \pm 0,29$ & $197,8 \pm 43,75$ & $4,37 \pm 1,02$ & $20,75 \pm 5,24$ & $1,62 \pm 0,99$ \\
& $p=N S$ & $p=N S$ & $p=N S$ & $p=N S$ & $p=N S$ & $p=0,0003^{* *}$ \\
\hline
\end{tabular}

* NS: no significativo, Anova. ** Leve vs otros grupos; PD: peritoneodiálisis, HD: hemodiálisis, Tx: trasplantados; T4T: T4 total, T4L: T4 libre, T3T: T3 total, T3L: T3 libre, TBG: Thyroid Biding Globulin, TSH: Hormona tirosestimulante 
Tabla 4. Test de TRH por \pm grupo $(\bar{x} \pm D S)$

\begin{tabular}{|c|c|c|c|c|c|c|}
\hline Grupos & $\begin{array}{c}\text { TSH } 0 \\
(\mathrm{uUl} / \mathrm{ml})\end{array}$ & $\begin{array}{l}\text { TSH } 30 \\
\text { (uUl/ml) }\end{array}$ & $\begin{array}{l}\text { TSH } 60 \\
(\mathrm{uUl} / \mathrm{ml})\end{array}$ & Delta 0 - 30 & Delta 0 - 60 & $\begin{array}{c}\text { DeltaTSH }_{\text {o-66 }} \times 100 \\
\text { Delta TSH 0-30 }\end{array}$ \\
\hline Leve & $4,25 \pm 0,95$ & $17,96 \pm 6,51$ & $15,25 \pm 5,95$ & 13,71 & 11,0 & $80,79(11,4)$ \\
\hline Moderado & $2,96 \pm 1,46$ & $9,82 \pm 4,6$ & $9,05 \pm 4,03$ & 6,86 & 6,09 & $91,02(12,7)$ \\
\hline PD & $2,4 \pm 1,4$ & $9,09 \pm 3,67$ & $8,40 \pm 4,50$ & 6,69 & 6,00 & $84,6 \quad(19,7)$ \\
\hline$H D$ & $1,99 \pm 1,19$ & $8,19 \pm 3,94$ & $7,65 \pm 3,86$ & 6,20 & 5,67 & $90,01(9,53)$ \\
\hline$T x$ & $1,63 \pm 0,99$ & $5,2 \pm 3,50$ & $3,91 \pm 2,7$ & 3,58 & 2,28 & $60,21 \quad(9,9)$ \\
\hline
\end{tabular}

PD: peritoneodiálisis, HD: hemodiálisis, Tx: trasplantados

Tabla 5. Tipos de respuesta al test de TRH según grupo

\begin{tabular}{lccccc}
\hline Respuesta & $\begin{array}{c}\text { Leve } \\
(\mathbf{\% )}\end{array}$ & $\begin{array}{c}\text { Moderada } \\
(\mathbf{\%})\end{array}$ & $\begin{array}{c}\text { PD } \\
(\mathbf{\%})\end{array}$ & $\begin{array}{c}\text { HD } \\
(\mathbf{\%})\end{array}$ & $\begin{array}{c}\text { TX } \\
(\%)\end{array}$ \\
\hline Normal & 0 & 10 & 30 & 0 & 30 \\
Exagerada & 10 & 0 & 0 & 0 & 0 \\
Prolongada & $80^{*}$ & $50^{*}$ & $50^{*}$ & $67^{*}$ & 0 \\
Retrasada & 0 & 10 & 0 & 0 & 0 \\
Deficiente & 0 & 30 & 20 & 33 & $60^{*}$ \\
Inclasificable & 10 & 0 & 0 & 0 & 10 \\
\hline
\end{tabular}

${ }^{*} p<0,001$, grupo $L$ vs demás grupos; ${ }^{\ddagger} p<0,001$, respuesta deficiente vs otras en grupo Tx, y Tx vs demás grupos.

pos se vio que la respuesta fue predominantemente prolongada en los grupos L $80 \%(8 / 10$ pacientes), M 50\% (5/10), PD 50\% (5/10) y HD 67\% (4/6), siendo el porcentaje de respuesta prolongada significativamente mayor en $\mathrm{L}$ que en los grupos M, PD, HD y TX $(p<0,01)$ (tablas 4 y 5). Sólo dos pacientes refirieron cefalea transitoria durante la administración del medicamento.

En el grupo TX, el tiempo promedio postrasplante fue de $31,7 \pm 22,4$ meses ( 11 a 66 meses), con promedio de creatinina de $0,88 \pm 0,19 \mathrm{mg} /$ dl. Todos se encontraban en tratamiento con prednisona (PRED), 7 con dosis diarias y 3 en días alternos. La dosis de mantención promedio fue de $0,09 \pm 0,03 \mathrm{mg} / \mathrm{kg}(0,05-0,17 \mathrm{mg} / \mathrm{kg})$, equivalentes a $11,61 \pm 3,41 \mathrm{mg} / \mathrm{m}^{2}$ de hidrocortisona. En relación a la respuesta al test de TRH en el grupo TX, se observó una respuesta deficiente en un $60 \%(6 / 10)$ de los pacientes, normal en $30 \%(3 / 10)$ y en $1 / 10$ fue inclasificable. La respuesta deficiente (delta TSH $<4,5$ ) fue significativamente más frecuente en TX que en los grupos L, M, HD y PD $(p<0,01)$ (tabla 5). Respecto del tratamiento inmunosupresor 6 pacientes se encontraban con ciclosporina (CSA) y micofenolato mofetil (MCF), 3 pacientes con tacrólimus (TAC) y MCF; y uno con azatriopina (AZA) más ciclosporina (CSA). Los 3 pacientes del grupo TX que presentaron una respuesta normal al test de $\mathrm{TRH}$, eran los que estaban en tratamiento con TAC, MCF y PRED en días alternos, a diferencia del resto que se trataba con CSA o AZA, MCF y PRED continua; sin embargo, el bajo número de pacientes no permite obtener diferencias estadísticamente significativas.

Tabla 6. Pacientes con alteraciones tiroideas o inclasificables

\begin{tabular}{|c|c|c|c|c|c|c|c|c|}
\hline & Grupos & $\begin{array}{c}\text { T3/T3L } \\
(\mu \mathrm{g} / \mathrm{dl}) / \\
(\mathrm{pg} / \mathrm{ml})\end{array}$ & $\begin{array}{l}\mathrm{T} 4 / \mathrm{T} 4 \mathrm{~L} \\
(\mu \mathrm{g} / \mathrm{dl})\end{array}$ & $\begin{array}{c}\text { TSH } 0 \\
(\mathrm{uUI} / \mathrm{ml})\end{array}$ & $\begin{array}{c}\text { TSH } 30 \\
(\mathrm{UUI} / \mathrm{ml})\end{array}$ & $\begin{array}{c}\text { TSH } 60 \\
(\mathrm{uUI} / \mathrm{ml})\end{array}$ & Delta 0 - 30 & Delta $0-60$ \\
\hline Paciente 1* & Leve & $178 / 6,1$ & $7,3 / 1,1$ & $7,67^{*}$ & 23,6 & 24,6 & 15,93 & 16,93 \\
\hline Paciente $2^{* *}$ & Leve & $176 / 5,2$ & $9,3 / 1,3$ & 5,08 & $31,6 * *$ & 27,9 & 26,52 & 22,8 \\
\hline Paciente 3 & $\mathrm{Tx}$ & $144 / 2,8$ & $12,7 / 1,9$ & $0,57 * * *$ & 1,29 & 0,89 & 0,891 & 0,719 \\
\hline
\end{tabular}

*Correspondiente a Hipotiroidismo primario subclínico, con Test de TRH inclasificables (sin delta TSH > $20 \mathrm{mUI} / \mathrm{ml}$; **Correspondiente a hipotiroidismo primario subclínico, con respuesta exagerada a TRH; ${ }^{* * *}$ Inclasificable por respuesta deficiente a TRH (sugerente de hipotiroidismo terciario) con T3T, T3L y T4L normales con T4 total elevada 
Se encontró una correlación positiva entre prolongación de la respuesta de TSH y los parámetros de función renal estudiados (creatininemia, BUN y clearance de creatinina) $(\mathrm{r}=$ $0,47, \mathrm{p}=0,001 ; \mathrm{r}=0,55, \mathrm{p}<0,001$ y $\mathrm{r}=-0,62$, $\mathrm{p}<0,001$, respectivamente), siendo estas correlaciones moderadas.

Hubo tres pacientes con alteraciones tiroideas o con perfil y respuesta inclasificable. Un paciente con hipotiroidismo primario subclínico, con respuesta exagerada a TRH; uno con hipotiroidismo primario subclínico, con test de TRH inclasificables ( $\sin$ delta TSH $>20$ ) y otro con TSH basal y frente a estímulo bajo con T4L y T3 total y libre normal (tabla 6).

\section{Discusión}

Los pacientes portadores de ERC presentan manifestaciones clínicas similares a las observadas en el hipotiroidismo, tales como talla baja, piel seca y fría, anorexia, letargia y constipación, por lo que es importante evaluar la función tiroidea en ellos. Estudios previos describen disminución de los niveles circulantes de hormonas tiroideas en $\operatorname{ERC}^{(1,2,3,11)}$ con valores normales de TSH basal en pacientes en tratamiento médico, manteniéndose alteradas en PD y $\mathrm{HD}$, con normalización post trasplante renal $^{3,11}$ hasta que el tratamiento inmunosupresor y corticoidal deteriora la funcionalidad normal del eje hipotálamo-hipofisiario-tiroideo.

En nuestro estudio tanto los valores basales de hormonas tiroideas como los de TBG estaban normales en todos los grupos, a diferencia de lo publicado en la literatura en población infantil con ERC en las décadas pasadas ${ }^{7,11}$; tampoco encontramos modificación de estos post trasplante. Estas diferencias pueden estar relacionadas con un mejor manejo de la ERC, así como también por el mejor estado nutritivo de nuestros pacientes. Los estudios publicados fueron realizados en la década de los 90, con pequeños números de pacientes donde la mayoría presentaban compromiso pondoestatural; distinto a lo encontrado en este estudio donde la mayoría se encuentran eutróficos y un alto porcentaje con sobrepeso u obesidad ${ }^{7,11}$. Actualmente un gran porcentaje de los pacientes tiene apoyo nutricional intensivo, a la vez se someten a programas de adecuación de diálisis periódicos que consideran el modelo de la cinética de la urea, con la medición regular de las dosis de diálisis (kt/v) y de acuerdo a valores preestablecidos, que les permiten una depuración y un balance nitrogenado más adecuado. Este mejor estado nutritivo y de adecuada sustitución renal, podría explicar esta situación.

La duración de la terapia dialítica puede influir también en los resultados. En nuestro grupo el tiempo promedio en diálisis no supera los 3 años porque los pacientes pediátricos se trasplantan hoy en día más precozmente que en aquellas épocas y que en adultos, lo que impide el deterioro que conllevaría la terapia dialítica prolongada. Se ha descrito mayor incidencia de bocio, especialmente en los pacientes sometidos a $\mathrm{HD}^{7,12,13}$; sin embargo, en el grupo HD estudiado, no se observó diferencias en los valores de hormonas tiroideas ni TBG, atribuibles a este factor; incluso en la única paciente que estaba en PD por largo tiempo (84 meses) sólo se observó una respuesta prolongada al test de TRH.

La respuesta al test de TRH en ERC L, M, PD y HD fue predominantemente prolongada, correlacionándose directamente con los niveles de creatinina y BUN, lo que demostraría un fenómeno adaptativo a nivel hipotálamico (terciario) frente a la progresión de la enfermedad renal independiente del tipo de tratamiento utilizado. Si bien en este estudio no contamos con grupo control, lo que es una debilidad, el número de pacientes estudiados en relación a los trabajos previamente publicados, da valor a estos hallazgos.

La mayoría de los pacientes trasplantados tenían valores basales normales de hormonas tiroideas con una respuesta deficiente o nula al test de TRH. Los tres pacientes trasplantados que presentaron una respuesta dentro de límites normales, correspondían a los que estaban en tratamiento con TAC, MCF y PRED en días alternos. El esquema inmunosupresor más potente en estos 3 pacientes, permite emplear dosis de corticoesteroides menores y a la vez, minimizar el riesgo de rechazo, tanto clínico como subclínico. Los valores normales de hormonas tiroideas encontrados en la mayoría de 
este grupo, pueden ser atribuidos, en parte, a que en este grupo estudiado se excluyó a los pacientes que tenían antecedentes de rechazo. Como lo describe la literatura, los episodios de rechazo agudo pueden producir alteraciones en el sistema endocrino, similares a los observados en pacientes con insuficiencia renal aguda o crónica $^{12}$.

La respuesta predominantemente deficiente o nula al test de TRH en TX podría estar relacionada con la dosis de corticoides y/o el esquema inmunosupresor utilizado, pues los 3 pacientes que recibían TAC, MCF y PRED en días alternos presentaron una respuesta normal a diferencia de los que recibían PRED en días contínuos u otro esquema inmunosupresor. Es sabido que los glucocorticoides suprimen la respuesta de TSH frente al estímulo de TRH y en el síndrome de Cushing está descrito una respuesta deficiente o nula a TRH, con valores de T3 y T4 bajos y falta de peak nocturno de $\mathrm{TSH}^{13}$. Nuestros pacientes se encontraban en tratamiento corticoidal con dosis fisiológicas de sustitución (equivalentes a $10-12 \mathrm{mg} / \mathrm{m}^{2} /$ día de hidrocortisona), lo que nos hace pensar que estos hallazgos podrían relacionarse más con el tipo de inmunosupresor que con el tratamiento corticoidal; sin embargo, el escaso número de pacientes no nos permite obtener conclusiones a este respecto.

Algunos autores ${ }^{3,6,7}$, afirman que existiría una normalización inicial de la respuesta al test de TRH post trasplante pero que posteriormente podrían ser influenciados tanto por el tratamiento corticoidal como por los otros inmunosupresores (CSA y AZA), que estarían jugando un rol en la inhibición de la respuesta. Debido al escaso número de pacientes TX de nuestro estudio, no podemos obtener conclusiones definitivas a este respecto.

Otro de los mecanismos señalados por algunos autores como responsables de las alteraciones tiroideas descritas en pacientes dializados, es la disminución de TBG, T4 y T3, secundario a pérdida por dializado ${ }^{4,10}$. Sin embargo, las pérdidas documentadas son pequeñas, por lo cual su reemplazo no se hace necesario ${ }^{7}$. En nuestro estudio TBG se encontraba normal y sin diferencias significativas con los otros grupos. Cabe señalar que estudios previos, utiliza- ban dosis de diálisis mayores no ajustadas por $\mathrm{kt} / \mathrm{v}$, lo que podría explicar mayor pérdida de hormonas tiroídeas en el dializado ${ }^{1,4,15}$.

En resumen, en este trabajo encontramos que los niveles basales de hormonas tiroideas estaban dentro de rango normal en los pacientes con ERC L, M, PD y HD; sin embargo, un alto porcentaje tenían respuesta prolongada a test de TRH en estos grupos, sugerente de trastorno terciario del eje que sería atribuible posiblemente a inhibidores urémicos, dada su correlación positiva con parámetros de función renal, como lo han descrito otros autores ${ }^{5,7}$. Diferente fue lo observado en pacientes trasplantados con buena función renal y sin rechazo, quienes presentaron predominantemente una respuesta deficiente o nula al test de TRH sugerente de una disfunción hipofisiaria, que podría estar relacionada al uso de corticoides y/o el tipo de inmunosupresores utilizados.

Estos hallazgos plantean un cambio en lo que debemos esperar de la función tiroidea basal en pacientes con ERC con los tratamientos nutricionales y dialíticos actuales: valores basales normales de hormonas tiroideas y tiroestimulante. El seguimiento de estos pacientes permitirá por otra parte, evaluar el valor predictivo del test de TRH sobre la función tiroidea futura de estos pacientes.

Finalmente, será necesario realizar nuevos estudios, que permitan aclarar cuál es el factor determinante en la presencia de respuesta normal a estimulación con TRH en los pacientes trasplantados en tratamiento con TAC y corticoides en días alternos, lo que permitiría proponer un tratamiento que no afectara la función tiroidea y por ende los efectos de ésta.

\section{Referencias}

1.- Kaptein EM: Thyroid hormone metabolism and thyroid diseases in chronic renal failure. Endocrine Reviews 1996; 17: 45-63.

2.- Mehta HJ, Joseph LJ, Desai KB, et al: Total and free thyroid hormone levels in chronic renal failure. J Postgrad Med 1991; 37: 79-83.

3.- Lim VS, Fang VS, Katz AL, Refetoff S: Thyroid function in chronic renal failure: a study of the pituitary-thyroid axis and peripheral turnover kinetics of thyroxine and triiodothyronine. J Clin Invest 1977; 60: 522-34. 
4.- Dusunsel R, Poyrazoglu HM, Gúndüz Z, Kurtoglu S, Kiris AL: Evidence of central hypothyroidism in children on continuous ambulatory peritoneal dialysis. Adv Perit Dial 1999; 15: 262-8.

5.- Pasqualini T, Zantiefer D, Balzaretti M: Evidence of hypothalamic-pituitary thyroid abnormalities in children with end stage renal disease. J Pediatr 1991; 118 : 873-8.

6.- Pasqualini T, Ferraris J, Fainstein-Day P: Hipothalamic-pituitary thyroid abnormalities in children after renal transplantation. Pediatric Nephrol 1996; 10 (5): 621-4.

7.- Lim V: Thyroid function in patients with chronic renal failure. Am J Kid Dis 2001; 38 suppl 1: s80-4.

8.- Schwartz GJ, Haycock GB, Edelmann CM Jr, Spitzer A: A simple estimate of glomerular filtration rate in children derived from body length and plasma creatinine. Pediatrics 1956; 58: 259-63.

9.- http://www.cdc.gov/nchs/about/major/nhanes/ growthcharts/clinical charts.htm

10.- Gruneiro De Papendieck L, Iorcansky S, Rivarola MA, Heinrich JJ, Bergada C: Patterns of ths response to trh in children with hypopituitarism. J Pediatr 1982;
100: 378-92.

11.- Cowden EA, Ractcliffe WA, Rattcliffe JG, Kennedy AC: Hypothalamic-pituitary function in uraemia. Acta Endocrinol 1981; 98: 488-95.

12.- Kebkowska U, Malyszko J, Mysliwiec M: Thyroid function and morphology in kidney transplant recipients, hemodialyzed, and peritoneally dialyzed patients. Transplant Proc 2003; 35: 2945-8

13.- Castellano M, Turconi A, Chaler E, Maceiras M, Rivarola Ma, Belgorosky A: Thyroid function and serum thyroid binding proteins in prepubertal and pubertal children with chronic renal insufficiency receiving conservative treatment, undergoing hemodialysis, or receiving care after renal transplantation. J Pediatr 1996; 128 (6): 778-790

14.- Bartalena L, Martino E, Petrini L, et al: The nocturnal serum thyrotropin surge is abolished in patients with adrenocorticotropin (acth)- dependent o acth independent cushing syndrome. J Clin Endocrinol Metab 1991; 72: 1195-9.

15.- Robey C, Shreedhar K, Batumann V: Effect of chronic peritoneal dialysis on thyroid function tests. Am J Kidney Dis 1989, 13: 99-103. 